\title{
MODELING OF PROPAGATION OF TRANSVERSE AND LONGITUDINAL ELECTROMAGNETIC WAVES IN NANOSTRUCTURES WITH NONLINEAR PROPERTIES
}

\author{
L. Uvarova ${ }^{1}$, Ya. Burenok ${ }^{2} \S$ \\ 1,2 Department of Applied Mathematics \\ Moscow state technological university "STANKIN" \\ 3a, Vadkovsky per., Moscow, 127055, RUSSIAN FEDERATION
}

\begin{abstract}
Interaction of an electromagnetic wave with nanostructure of a cylindrical form with nonlinear dependence of dielectric permeability on a field is considered. The mathematical model and algorithm of finding of a longitudinal electromagnetic wave considering influence of a transverse wave and contribution of potential of interaction near a singular point of system is offered. On the basis of the developed algorithm numerical results for a metal nanocluster were received.
\end{abstract}

AMS Subject Classification: 82D 80

Key Words: Schrödinger equation, nonlinear Schrödinger equation, Mie theory, nanostructures, transverse and longitudinal waves

\section{Introduction}

Recently there is a growing interest in meso- and nanoparticles. The main reason for this is that these particles have unique chemical and physical properties, which an be regulated by changing the composition, atomic ordering, particle size. More and more the quantum properties of particles are manifested than the solid-state structures are approach to nanostructures area [1], [2], [3], [4]. Particularly, metal cluster[5] perspectively discover creating new unique switch-

Received:

Revised:

August 25, 2016

Published: September 26, 2016

September 30, 2016 (c) 2016 Academic Publications, Ltd. url: www.acadpubl.eu

${ }^{\S}$ Correspondence author 
ing, memory and augmentative elements for information systems, and help to improve the accuracy and workability of manufacture of composite materials for creating ultramodern optical instruments. Therefore, it seems urgent to study the interaction of electromagnetic radiation with metal nanoparticles due the implementation of management of capabilities in various nanosystems. It's known, by revising interaction of electromagnetic waves with small particles except the transverse, formed and longitudinal electromagnetic waves too. That's why, due to active researching in meso- and nanosystems area, that happens currently in the world, it can be actual to consider the type of propagation longitudinal electromagnetic waves in same structures. For their description can be used similar algorithm [6]:

1. The solution of problem spreading transverse electromagnetic waves in a certain geometric structure. This solution can be obtained based on Mie theory and his generalization.

2. Schrödinger equation solution taking into account the interaction potential and the amplitude of the electric and magnetic(in general) vectors, found in step 1.

3. Polarization vector definition using Psi-functions, found in step 2.

4. Definition of new values the electric and magnetic vectors, using polarization vector.

We have considered interactive with the system of simulated cylinder in this article. The dielectric permittivity of the cylindrical particles depends of frequency, number of molecules (to a certain size of the nanoparticle) in nanosystems and current position and a quadratic dependence on a field:

$$
\varepsilon=\varepsilon_{0}+\alpha|\mathbf{E}|^{2}
$$

where is $\alpha$ is a nonlinearity parameter, $\varepsilon_{0}$ is a permittivity in the absence of a electromagnetic field.

\section{Propagation Modeling of Transverse Electromagnetic Waves}

Consider a fall of monochromatic plane-polarised wave in the cylinder there electric and magnetic vector is harmonic time. 


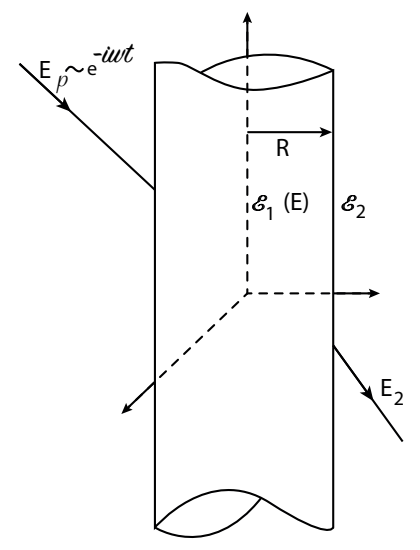

Figure 1: Interaction of a monochromatic wave with cylinder

On the basis of approach developed in [8], [9] at the first step we get obtain the equations for determining time-independent vectors and from Maxwell's equations:

$$
\nabla \times \mathbf{E}_{i}=k_{2} \mathbf{H}_{i}, \nabla \times \mathbf{H}_{i}=k_{1} i \mathbf{E}_{i}, \nabla \mathbf{D}_{i}=0, \nabla \mathbf{B}_{i}=0
$$

where $k_{1 i}=i \omega\left(\varepsilon_{i}+i 4 \pi \sigma_{i} / \omega\right) / c, k_{2}=i \omega / c, k^{(i)}=-k_{2} k_{1 i}, \sigma_{i}$ is the conductivity of the medium, $c$ is the velocity of light, $i=1$ is a cylinder, $i=2$ non-conductive medium. Properly from expression (1) the quantity $k_{i}$ depends on the electric vector.

Assume that $\mathbf{E}=\mathbf{E}^{e}+\mathbf{E}^{m}, \mathbf{H}=\mathbf{H}^{e}+\mathbf{H}^{m}, E_{z}^{e}=E_{z}, H_{z}^{m}=H_{z}, E_{z}^{m}=0$, $H_{z}^{e}=0$. Then we get the equation (2) for the scalar Debye potentials [8], [10] that will help determine the components of vectors $\mathbf{E}$ and $\mathbf{H}$ :

$$
\begin{gathered}
\nabla\left(k \nabla \Pi^{e}\right)+k^{2} \tilde{k} \iint \tilde{k} \frac{\partial^{2} \Pi^{e}}{\partial z \partial \varphi}-\frac{\partial \tilde{k}}{\partial z} \frac{\partial \Pi^{e}}{\partial z}- \\
-\frac{1}{r} \frac{\partial}{\partial r}\left(r \int \frac{\partial \tilde{k}}{\partial z} \frac{\partial \Pi^{e}}{\partial r} \partial r\right)-\frac{1}{r^{2}} \frac{\partial}{\partial \varphi}\left(\int \frac{\partial \tilde{k}}{\partial z} \frac{\partial \Pi^{e}}{\partial \varphi} \partial z\right)=0 \\
\nabla \Pi^{m}+k^{2} \tilde{k} \Pi^{m}-k^{2} \tilde{k} \int \Pi^{m} \frac{\partial \tilde{k}}{\partial \varphi} \partial \phi=0 \\
H_{r}=H_{r}^{e}+H_{m}^{e}=-\frac{k_{11}^{0}}{r} \int \tilde{k} \frac{\partial^{2} \Pi^{e}}{\partial z \partial \varphi} \partial z+\frac{\partial^{2} \Pi^{m}}{\partial r \partial z}
\end{gathered}
$$




$$
\begin{gathered}
H_{z}=-\frac{1}{r} \frac{\partial}{\partial r}\left(r \frac{\partial^{2} \Pi^{m}}{\partial r \partial z}\right)-\frac{1}{r^{2}} \frac{\partial^{2} \Pi^{m}}{\partial \varphi^{2}} \\
H_{\varphi}=k_{11}^{0} \int \tilde{k} \frac{\partial^{2} \Pi^{e}}{\partial r \partial z} \partial z+\frac{1}{r} \frac{\partial^{2} \Pi^{m}}{\partial \varphi \partial z}, \\
E_{r}=\frac{\partial^{2} \Pi^{e}}{\partial r \partial z}+\frac{k_{2}}{r} \frac{\partial \Pi^{m}}{\partial \varphi}, \\
+\frac{1}{\tilde{k} r}\left[\frac{\partial}{\partial r}\left(r \tilde{k} \frac{\partial \Pi^{e}}{\partial r}\right)+\frac{1}{r} \frac{\partial}{\partial \varphi}\left(\tilde{k} \frac{\partial \Pi^{e}}{\partial \varphi}\right)\right]+ \\
\left.\left.E_{z}\left[\frac{\partial \tilde{k}}{\partial r} \partial z\right)+\frac{1}{r} \frac{\partial}{\partial \varphi} \int \frac{\partial \tilde{k}}{\partial z} \frac{\partial \Pi^{e}}{\partial \varphi} \partial z\right)\right] \\
E_{\varphi}=\frac{1}{r} \frac{\partial^{2} \Pi^{e}}{\partial z \partial \varphi}+k_{2} \frac{\partial \Pi^{m}}{\partial r},
\end{gathered}
$$

where $\tilde{k}=\varepsilon / \varepsilon_{0}, k_{11}^{2}=i \omega \varepsilon_{0} / c$.

For realization (2)-(4) and (5)-(6) requires performance of one of the following conditions for transverse field:

$$
\frac{\partial \tilde{k}}{\partial \varphi}=0, \frac{\partial \tilde{k}}{\partial r}=0, E_{\varphi}^{e} E_{\varphi}^{m}+E_{r}^{e} E_{r}^{m}=0
$$

At the interface of two media $(r=R)$ the equality condition for the tangential components of the electric and magnetic vectors must be satisfied:

$$
\begin{aligned}
E_{p z}(R)+E_{s z}(R) & =E_{1 z}(R), E_{p \varphi}+E_{s \varphi}(R)=E_{1 \varphi}(R), \\
H_{p z}(R)+H_{s z}(R) & =H_{1 z}(R), H_{p \varphi}+H_{s \varphi}(R)=H_{1 \varphi}(R),
\end{aligned}
$$

there index $p$ denotes the incident wave and the index $s$ denotes the scattered wave. Let us find asymptotic solutions for the electric vector of the transverse field. For this we consider two main directions of the ray propagation: along the radial coordinate $r$ and along the coordinate $z$ and assume that the following inequalities are fulfilled: $\left|E_{z}\right|>>\left|E_{\varphi}\right|,\left|E_{z}\right|>>\left|E_{r}\right|$. We believe that the contribution $|\mathbf{E}|^{2}$ into the value of the dielectric permittivity is not very large, that allows consider the nonlinearity measure $h=\max _{r}|\alpha| \cdot|\mathbf{E}|^{2} \varepsilon_{0}^{-1}$ as a small parameter and enter the type of slow variables $\tilde{y}=y h$. 


\subsection{Propagation of a Ray Along Coordinate $r$}

If the ray propagates along the coordinates $r$ it is possible to write for potential $\Pi^{e}=\psi(r, \varphi) \exp (i k r)$. Introduce the slow variable $\chi=r h^{2}$. Then the following equation arises from $(3)$ at limitation $h^{2}$ :

$$
i\left(\frac{\partial \tilde{\psi}}{\partial \chi}+\frac{\tilde{\psi}}{2 \tilde{\chi}}\right)+\frac{1}{2 \tilde{\chi}^{2}} \frac{\partial^{2} \tilde{\psi}}{\partial \tilde{\varphi}^{2}} \mp|\tilde{\psi}|^{2} \tilde{\psi}=0,
$$

where $\tilde{\psi}=\left(k^{(1)}\right)^{2} \sqrt{\alpha / \varepsilon_{0}} \psi / h, \tilde{\chi}=\chi k^{(1)}, \tilde{\varphi}=\varphi / h$. The substitution: $\tilde{\psi}=$ $\psi_{0} \exp \left(i m^{2} / 2 \tilde{\chi}+i m \tilde{\varphi}\right) / \sqrt{\tilde{\chi}}$ comes the last equation to next form:

$$
\frac{i \partial \psi_{0}}{\partial \tilde{\chi}} \pm \frac{1}{2} \frac{\left|\psi_{0}\right|^{2} \psi}{\tilde{\chi}}=0 .
$$

Then the expression for the electric Debye potential and can write in the view:

$$
\begin{gathered}
\Pi^{2}=\Gamma_{e} \sqrt{\frac{\varepsilon_{0}}{\alpha}}\left(\left(k^{(1)}\right)^{2} \sqrt{k^{(1)} r}\right)^{-1} \times \\
\times \exp \left[i\left( \pm 0,5\left|\Gamma_{e}\right|^{2} \ln \left(h^{2} k^{(1)} r\right)+\frac{m^{2}}{2 k^{(1)} r h^{2}}+k^{(1)} k+\frac{m \varphi}{h}\right)\right], \\
E_{z}=\frac{\Gamma_{e} \sqrt{\frac{\varepsilon_{0}}{\alpha k(1) r} \exp \left(i\left( \pm 0,5\left|\Gamma_{e}\right|^{2} \ln \left(h^{2} k^{(1)} r\right)+\frac{m^{2}}{2 k^{(1)} r h^{2}}+k^{(1)} k+\frac{m \varphi}{h}\right)\right)},}{}
\end{gathered}
$$

Here $m, \Gamma_{e}$ are constants.

\subsection{Propagation of a Ray Along Coordinate $z$}

Other solution arises at the consideration of the propagation of a ray along coordinate $z$. Then the electric potential is written as $\Pi^{e}=\psi(r, z) \exp \left(i k^{(1)} z\right)$. In this case the modification of the nonlinear Schrödinger arises from the equation (3) at limitation $h^{3}$ :

$$
\frac{\partial^{2} \tilde{\psi}}{\partial \tilde{z}^{2}}+i \frac{\partial \tilde{\psi}}{\partial \tilde{r}}+i \frac{\tilde{\psi}}{\tilde{r}} \pm 2|\tilde{\psi}|^{2} \tilde{\psi}=0,
$$

The substitutions from (5): $\tilde{\psi}=(1 / r) \exp \left(i \tilde{z}^{2} / 4 \tilde{r}\right) \Psi(x, \zeta), x=\tilde{z} / \tilde{r}, \zeta=-1 / \tilde{r}$ transform it to the classical NLS:

$$
i \Psi_{0 \zeta}+\Psi_{0 x x} \pm 2|\Psi|^{2} \Psi_{0}=0 .
$$


Equation (12) has as well soliton solution depending on the sign of "+" or "-":

$$
\begin{aligned}
\Psi_{0 \zeta}^{(+)} & =A_{0} \exp \left[i\left((v x / 2)-\left(\left(v^{2} / 2\right)-A_{0}^{2}\right) \zeta+\theta_{0}\right)\right] \\
& \cdot \operatorname{sech}\left(A_{0}\left(x-v \zeta-x_{0}\right)\right) \\
\Psi_{0 \zeta}^{(-)} & =A_{0} \exp \left[i\left((v x / 2)-\left(\left(v^{2} / 4\right)+2 A_{0}^{2}\right) \zeta+\theta_{0}\right)\right] . \\
& \cdot \operatorname{th}\left(A_{0}\left(x-v \zeta-x_{0}\right)\right),
\end{aligned}
$$

where $A_{0}$ is the amplitude, $v$ is the group velocity of the solitons, and $x_{0}, \theta_{0}$ are constants.

For electric potential $\Pi^{e}$, we obtain the solution, which meets the conditions for self-focusing of a ray:

$$
\begin{aligned}
\Pi_{s}^{e+} & =2{\frac{2 \varepsilon_{0}}{\alpha}}^{0,5} \frac{B_{0}}{r k^{(1)}} \exp \left[i \left(k^{(1)} r+\frac{z^{2} k^{(1)}}{2 r}+\frac{v_{0} z}{r}+\right.\right. \\
& \left.\left.+\left(\frac{v_{0}^{2}}{4}-B_{0}^{2}\right) \frac{2}{k^{(1)} r}+\theta\right)\right] \operatorname{sech}\left(2 B_{0}\left(\frac{z}{r}+\frac{v_{0}}{2 k(1) r}\right)\right),
\end{aligned}
$$

where $B_{0}, v_{0}, \theta_{0}$ are constants and $x_{0}$ due to the symmetry of the problem.

The component $E_{z}$ in the first approximation can be considered proportional to $\Pi^{2}$.

From (15) it follows that along coordinate $z$ the solution is a the breather with top $z^{*}=-v_{0} / k(1)$.

To the sign "-" of the equation (12) satisfies the following decision:

$$
\begin{aligned}
\Pi_{s}^{e-} & =2{\frac{2 \varepsilon_{0}}{\alpha}}^{0,5} \frac{B_{0}}{r k^{(1)}} \exp \left[i \left(k^{(1)} r+\frac{z^{2} k^{(1)}}{2 r}+\frac{v_{0} z}{r}+\right.\right. \\
& \left.\left.+\left(\frac{v_{0}^{2}}{4}+2 B_{0}^{2}\right) \frac{2}{k^{(1)} r}+\theta\right)\right] \operatorname{th}\left(2 B_{0}\left(\frac{z}{r}+\frac{v_{0}}{2 k(1) r}\right)\right),
\end{aligned}
$$

\section{Schrödinger Equation. The Longitudinal Wave}

By the method described above for definition of an electric vector of a transverse electromagnetic field the nonlinear equation of Schrödinger was solved. The received soliton solutions it is used further for definition of the field considering longitudinal waves. For this purpose we will use Maxwell-Schrödinger's model described, for example, in [6], [7] and we will come to the following system: 


$$
\begin{gathered}
\left.\left(T-i \mathbf{A}\left(\mathbf{r}^{\prime}\right)\right) \cdot \nabla_{r} / c+\left\|\mathbf{A}\left(\mathbf{r}^{\prime}\right)\right\|^{2} c^{2}+V(\mathbf{r})\right) \psi_{s h}=E_{t o t} \psi_{s h} \\
\mathbf{P}\left(\mathbf{r}^{\prime}\right)=-n\left(\mathbf{r}^{\prime}\right) \int \psi_{s h} \mathbf{r} \psi_{s h}^{*} \mathbf{r}^{\prime} d r \\
\nabla \cdot \mathbf{E}=-4 \pi \mathbf{P}\left(\mathbf{r}^{\prime}\right)
\end{gathered}
$$

where $\mathbf{r}^{\prime}$ is spatial coordinate for Maxwell's, $\mathbf{r}$ is spatial coordinate for the Schrödinger equation, A vector potential, $n\left(\mathbf{r}^{\prime}\right)$ is concentration of the electron gas, $T$ is the total kinetic energy, $V(\mathbf{r})$ is the potential interaction of the system, $E_{t o t}$ is the total energy. We will consider the time-independent Schrödinger equation as we assume, as $\mathbf{E}$ and $\mathbf{H}$ change under the harmonious law.

From (17), using substitution from [7], [11] it is possible to receive the equation:

$$
\left(T-\mathbf{r E}\left(\mathbf{r}^{\prime}\right)+V(\mathbf{r})\right) \psi_{s h}=E_{t o t} \psi_{s h}
$$

where $\mathbf{E}\left(\mathbf{r}^{\prime}\right)$ is electric vector of the external field.

We can write down the equation (17) for the particle consisting of several atoms. Schrödinger equation will look like this [12], [13]:

$$
\begin{aligned}
(-(\hbar /(2 m)) & \sum_{i} \nabla_{i}^{2}-(1 / 2) \sum_{j} \hbar^{2} \nabla_{j}^{2} /\left(2 M_{j}\right)+ \\
& +(1 / 2) \sum_{i \neq k} e^{2} / r_{i k}+(1 / 2) \sum_{J \neq K} Z_{J} Z_{K} e^{2} / R_{J K}- \\
& \left.-\sum_{J i} Z_{j} e^{2} / r i j+\mathbf{r E}\left(\mathbf{r}^{\prime}\right)\right) \psi_{s h}=E_{t o t} \psi_{s h}
\end{aligned}
$$

$r_{i j}=\left|r_{i}-r_{k}\right|, R_{J L}=\left|R_{J}-R_{K}\right|, r_{i j}=\left|r_{i}-R_{j}\right|$

$$
H=\left(-(\hbar /(2 m)) \sum_{i} \nabla_{i}^{2}-(1 / 2) \sum_{j} \hbar^{2} \nabla_{j}^{2} /\left(2 M_{j}\right)+(1 / 2) \sum_{i \neq k} e^{2} / r_{i k}+\right.
$$
$(1 / 2) \sum_{J \neq K} Z_{J} Z_{K} e^{2} / R_{J K}-\sum_{J i} Z_{j} e^{2} / r i j$ is Hamilton function of the system, $m$ is the electron mass, $M$ is the positively charged ion mass, $Z$ is the ion charge.

Thus it is necessary take into account the kinetic energy of all the electrons and nuclei of the system for solution. Even for the elementary multielectron systems the exact solution of the equation of Schrödinger does not manage to be found and therefore it is necessary to apply various ways of his approximate decision. Consider the possible of algorithm for describing the task. 


\subsection{Without External Influence}

For a start we will consider a task without taking influence on system of transverse electromagnetic field:

$$
H \Psi_{s h}=E \Psi_{s h}
$$

Simplify an expression (22). One of the main method of describing metallic nanostructures including clusters is a jellium model ([13], [15]), there particle is divided on two subsystems: positive ions of the core and delocalized valence electron system.

The valence electrons are moving in the field formed by clusters ions, which in first approximation can be replaced by average background uncompensated charge. In the simplest case, we consider that the ionic core represents evenly the charged sphere[16]. Then the potential $V_{\text {core }}$ will be written as:

$$
V_{\text {core }}= \begin{cases}\frac{N e^{2}}{2 R}\left(3-\left(r_{i} / R\right)^{2}\right), & r_{i} \leq R \\ \frac{N e^{2}}{r_{\mathrm{el}}}, & r_{i}>R\end{cases}
$$

there $N e$ is full charge of the sphere, $R$ is the radius of the ion core (being the only macroscopic parameter of the model) and $R=r_{s} N^{(1 / 3)}, r_{s}$ is average distance between atoms in bulk metal.

Use the Born-Oppenheimer approximation[14] and divide the task on two: the movement of electrons in the field of motionless ions and the movement bark without volume propagation of electrons. Such view is proved, using adiabatic approach [7], [17]. As ions it is much heavier than electrons, ions slowly react to change of electronic concentration whereas electrons are adiabatically reconstructed at change of provision of ions. Then wave function of (22) can be written as

$$
\Psi_{s h}(r, R)=\Phi(r, R) \psi(R)
$$

where $\Phi(r, R)$ is full function of valance electronic system, $r$ is the spatial coordinates of the electrons and $\psi(R)$ is physical wave function of the ion core.

Then the equation (21) is developed on two:

$$
\begin{gathered}
H_{e l}(r, R) \Phi(r, R)=E_{e l} \Phi(r, R), \\
\left(-\hbar^{2} \frac{1}{2 M} \frac{d^{2} \psi(R)}{d R^{2}}+E\right) \psi(R)=\epsilon \psi(R),
\end{gathered}
$$

where $H_{e l}(r, R)$ is the Hamilton function of the electron system. $E, E_{e l}, \epsilon$ are the total energy, self-energy of the electron system and the vibrations of the ion core. $M=(3 / 5) \sum^{N} M_{a t}$ is reduced mass of the core, $M_{a t}$ is mass of each ion. 
Consider $R$ as an independent parameter, and for fixed $R$ we solve the equation (25).

Many-electron function $\Phi(r, R)$ can be represented by a single Slater determinant of the normal one-electron functions [18].

$$
\begin{aligned}
& \Phi\left(r_{1}, r_{2}, \ldots, r_{N}, \alpha, \beta, \ldots, v\right)= \\
& =\frac{1}{\sqrt{N !}}\left|\begin{array}{cccc}
\phi_{\alpha}\left(r_{1}\right) & \phi_{\alpha}\left(r_{2}\right) & \ldots & \phi_{\alpha}\left(r_{N}\right) \\
\phi_{\beta}\left(r_{1}\right) & \phi_{\beta}\left(r_{2}\right) & \ldots & \phi_{\beta}\left(r_{N}\right) \\
\vdots & \vdots & \vdots & \vdots \\
\phi_{v}\left(r_{1}\right) & \phi_{v}\left(r_{2}\right) & \ldots & \phi_{v}\left(r_{N}\right) .
\end{array}\right|
\end{aligned}
$$

Here the variables $r_{i}$ include the coordinates of spin and space of particle $i$. The quantum numbers $\alpha, \beta, \ldots, v$ encompass all possible quantum numbers needed to specify a particular system. We can define one-electron functions $\phi_{i}$ and energies of valence electrons as solutions of the Hartree-Fock self-consistent equations [17], [20],[19]:

$$
\begin{aligned}
\left(-\hbar^{2} \nabla_{i}^{2} /(2 m)\right. & -e V_{\text {core }}(r) \phi_{i}\left(\mathbf{r}_{i}\right)- \\
& -\sum_{j=1}^{N} \int \phi_{j}^{*}\left(\mathbf{r}_{j}\right) \frac{e^{2}}{\left|\mathbf{r}_{j}-\mathbf{r}_{i}\right|}\left(\phi_{i}\left(\mathbf{r}_{i}\right) \phi_{j}\left(\mathbf{r}_{j}\right)-\right. \\
& \left.-\phi_{i}\left(\mathbf{r}_{j}\right) \phi_{j}\left(\mathbf{r}_{i}\right)\right) d \mathbf{r}_{j}=E_{i} \phi_{i}\left(\mathbf{r}_{i}\right), \quad 1 \leq i \leq N
\end{aligned}
$$

Thus we will consider a system from $\mathrm{N}$ equations for $\mathrm{N}$ one-electron states, which in atomic units will take a form:

$$
\begin{aligned}
\left(-\nabla_{i}^{2} /(2 m)\right. & -V_{\text {core }}(r) \phi_{i}\left(\mathbf{r}_{i}\right)- \\
& \left(\sum_{j=1}^{N} \int \phi_{j}^{*}\left(\mathbf{r}_{j}\right) \frac{1}{\left|\mathbf{r}_{j}-\mathbf{r}_{i}\right|} \phi_{j}\left(\mathbf{r}_{j}\right) d \mathbf{r}_{j}\right) \phi_{i}\left(\mathbf{r}_{i}\right)- \\
& -\sum_{j}^{N}\left(\int \phi_{j}^{*}\left(\mathbf{r}_{j}\right) \frac{1}{\left|\mathbf{r}_{j}-\mathbf{r}_{i}\right|} \phi_{i}\left(\mathbf{r}_{j}\right) d \mathbf{r}_{j}\right) \phi_{j}\left(\mathbf{r}_{i}\right)= \\
& =E_{i} \phi_{i}\left(\mathbf{r}_{i}\right), \quad 1 \leq i \leq N,
\end{aligned}
$$

where the terms of the first sum express the total electrostatic potential of electrons:

$$
U_{e l} \mathbf{r}=\sum_{j=1}^{N} \int \phi_{j}^{*}(\mathbf{s}) \frac{1}{|\mathbf{r}-\mathbf{s}|} \phi_{j}(\mathbf{s}) d(\mathbf{s}) .
$$


Equestion (28) differentially has an appearance:

$$
\nabla U_{e l} \mathbf{r}=-4 \pi \sum_{j=1}^{N} \phi_{j}^{*} \phi_{j}
$$

Each term of the second sum determines the exchange potential which arises because the electron doesn't interact with itself:

$$
\begin{gathered}
U_{i j} \mathbf{r}=\int \phi_{j}^{*}(\mathbf{s}) \frac{1}{|\mathbf{r}-\mathbf{s}|} \phi_{i}(\mathbf{s}) d(\mathbf{s}) . \\
\nabla U_{i j} \mathbf{r}=-4 \pi \phi_{j}^{*} \phi_{i}
\end{gathered}
$$

For the solution of the equations of Hartree-Fock in the central-field approximation it will be useful to pass to spherical coordinates. One-electron wave function can be presented as a product of radial, angular and spin components [17], [18]:

$$
\phi_{i}(x)=\frac{1}{r} R_{n l}(r) Y_{l m}(\varphi, \theta) X_{\sigma},
$$

there $x=(r, \sigma), n$ is the principal quantum number, $l$ is the orbital number, $m$ is the magnetic quantum number. By analogy with atoms a set of electronic states with identical $n$ and $l$ form a $n l$-subshell.

In expression (30) is angular part of wave function, which can be presented as $[21]$ :

$$
Y_{l m}(\varphi, \theta)=\sqrt{\frac{2 l+1}{4 \pi} \cdot \frac{(l-m) !}{(l+m) !}} P_{l}^{m}(\cos \theta) \exp (i m \varphi)
$$

where $P_{l}^{m}(\cos \theta)$ is the Legendre polynomials. For definition of radial component $R_{n l}(r)$ we will write down exchange potential as:

$$
\left(\frac{1}{r} \frac{\partial^{2}}{\partial r^{2}}+\frac{1}{r} \nabla_{(\theta \varphi)}\right) r U_{n l, n^{\prime} l^{\prime}}=4 \pi R_{n l} R_{n^{\prime} l^{\prime}} Y_{l^{\prime} m^{\prime}}^{*} Y_{l m}
$$

This expression can be simplified when using an addition theorem: $P_{l}(\cos \gamma)=$ $\frac{4 \pi}{2 l+1} \sum_{m=-l}^{l} Y_{l m}^{*}\left(\theta^{\prime} \varphi^{\prime}\right) Y_{l m}(\theta \varphi)$. Thus the electronic potentials making selfcontained subshell can be found from following equation:

$$
\left(\frac{1}{r} \frac{d^{2}}{d r^{2}}-\frac{k(k+1)}{r^{2}}\right) U_{n l, n^{\prime} l^{\prime}}(r)=(2 k+1) R_{n l} R_{n^{\prime} l^{\prime}}
$$


for each $n, l, n^{\prime}, l^{\prime}$ and $k, 0 \leq k \leq l+l^{\prime}$. Then for receiving a radial component equestion of Hartree-Fock will be solved:

$$
\begin{gathered}
\left(\frac{1}{r} \frac{d^{2}}{d r^{2}}-\frac{l(l+1)}{r^{2}}-2\left(U_{\text {core }}(r)-\sum_{n^{\prime} l^{\prime}} 2\left(2 l^{\prime}+1\right) \hat{U}_{n^{\prime} l^{\prime}, n^{\prime} l^{\prime}, 0}\right)+\right. \\
\left.+2 \epsilon_{n l}\right) R_{n l}=2 \sum_{n^{\prime} l^{\prime}} \sum_{k=0}^{l+l^{\prime}} \beta_{l l^{\prime} k} \hat{U}_{n^{\prime} l^{\prime}, n^{\prime} l^{\prime}, 0} R_{n^{\prime} l^{\prime}}
\end{gathered}
$$

there $\sum_{n^{\prime} l^{\prime}} 2\left(2 l^{\prime}+1\right) \hat{U}_{n^{\prime} l^{\prime}, n^{\prime} l^{\prime}, 0}$ is total potential of all electrons in the each $n^{\prime} l^{\prime}$ shell. Thus the system (27)-(29) passes into a system of ordinary differential equestions which can be written in matrix form:

$$
\begin{gathered}
\left(H_{i}+2 \epsilon_{i}\right) R_{i}=\sum_{i} h_{i j} R_{j}, \quad 1 \leq i \leq m, \\
L_{k} \hat{U}_{i j k}=R_{i} R_{j} \text { for each } i, j \text { and } k
\end{gathered}
$$

where $H_{i}=\left(\frac{1}{r} \frac{d^{2}}{d r^{2}}-\frac{l(l+1)}{r^{2}}-2\left(U_{\text {core }}(r)-\sum_{n^{\prime} l^{\prime}} 2\left(2 l^{\prime}+1\right) \hat{U}_{n^{\prime} l^{\prime}, n^{\prime} l^{\prime}, 0}\right)+2 \epsilon_{n l}\right)$, $h_{i j}=2 \sum_{n^{\prime} l^{\prime}} \sum_{k=0}^{l+l^{\prime}} \beta_{l l^{\prime} k} \hat{U}_{n^{\prime} l^{\prime}, n^{\prime} l^{\prime}, 0}$ and $L_{k}=\left(\frac{1}{r} \frac{d^{2}}{d r^{2}}-\frac{k(k+1)}{r^{2}}\right) \frac{1}{2 k+1}$. The radial function satisfies the boundary conditions:

$$
R_{i}(0)=0, R_{i}(r) \rightarrow 0 \text { if } r \rightarrow \infty
$$

And conditions of a normalization:

$$
\int_{0}^{\infty} R_{i}^{2}(r) r^{2} d r=1
$$

$R_{i}$ belongs to a separate $n l$-shell.

For finding of solutions of the equations (33)-(37) an iterative method are used [21]:

1. On the $p$-th step the radial functions $R_{i}$ and corresponding values of $\epsilon_{i}$ are known (on the first step ( $\mathrm{p}=0)$ we choose a set of suitable functions).

2. Total potentials and exchange potentials are found from the expressions: $L_{k} \hat{U}_{i j k}^{(p)}=R_{i}^{(p)} R_{j}^{(p)}$ by tridiagonal matrix algorithm.

3. $H_{i}^{(p)}$ and $h_{(p)}$ are based on potentials. 
4. New radial functions is a solve of the Schrödinger equations:

$$
\left(H_{i}^{(p)}+2 \epsilon_{i}^{(p)}\right) R_{i}(p+1)=\sum_{j} h_{i j}(p) R_{j}^{(p)}
$$

and conditions of a normalization.

As a result we will receive the vector of values at the given $R$.

\subsection{The Wave Function Taking into Account the External Field}

Now consider the wave function under the action of the particles of the external electromagnetic field and expand it into a series of function:

$$
\psi_{s h}(\mathbf{r}, R)=\sum_{i} a_{n}\left(\mathbf{E}_{0}\right) \mathbf{r} \mathbf{E} \Psi_{s h}(\mathbf{r}, R),
$$

where coefficient $a_{n}$ depends of an amplitude of the incident wave.

Now substitute $\psi_{s h}(\mathbf{r}, R)$ at the equation (18):

$$
\mathbf{P}\left(\mathbf{r}^{\prime}\right)=-n\left(\mathbf{r}^{\prime}\right) \int \psi_{s h} \mathbf{r} \psi_{s h}^{*} \mathbf{r}^{\prime} d r
$$

After that, solving Poisson equation $\nabla \cdot \mathbf{E}=-4 \pi \mathbf{P}\left(\mathbf{r}^{\prime}\right)$, we obtained the values of the vector of the longitudinal field. Then absolute value of the total electric vector amplitude s equal to the sum of the longitudinal and transverse fields.

\section{Conducting of Numerical Experiments}

The program for carrying out calculations with cylindrical particle of lithium in an electromagnetic field by the formed ray of the solid state laser with a frequency of radiation about $10^{15} \mathrm{~Hz}$, was developed. The program is realized in the environment of Matlab R2015a as it possesses enough the firmware functions, facilitating creation of a program code. At the first stage by means of Maxwell's equations and modification of a non-linear Schrödinger equation we found an electric vector of a transverse electromagnetic field in Maxwell domain $\Omega$. The received value it was used for calculation of longitudinal waves in a subdomain $\Omega_{i} \in \Omega$.

Results of the made computing experiment are given below. Let's consider a case of distribution of the ray along radial coordinate. In the figures (2) 
and (3) the dependency graphs of the square of absolute values of the full electric vector amplitude from the frequency of an incident radiation and the same but for the vector including only a transverse component of an electric field are constructed. We considered particles with radiuses of 2 (2) and of 1 (3) nanometers. It is visible that at the figure (2) the structure of graphs is more similar, than at the figure (3), where distinctions between curves are shown more obviously. Perhaps, it is bound to increase in influence of quantum properties in particles of the smaller size.

Let's consider for particles of the same size a case when the direction of the ray coincides with the direction of an axis $z$. At positive parameter $\alpha>0$ (fig. (4), (5)) we see the strong structural distinction of a curve of the complete electric vector about the curve describing only a transverse wave. And as in the previous case, distinctions amplify with decrease of radius.

Such tendency will remain also whith the negative parameterr $\alpha<0$ (figures (6), (7)) though structurally schedules become more similar. However it is worth noticing that unlike the previous cases, the curve describing the transversal field becomes above a curve of the full electric vector.

\section{Conclusions}

Thus, in this article the problem of interaction of an electromagnetic wave with a nanoparticle of a cylindrical form and emergence of longitudinal waves was considered. Two cases of distribution of the ray were considered: along the radial coordinate $r$ and along the coordinate $z$. The mathematical model and algorithm for finding of longitudinal waves taking into account the size of a transverse wave and interaction potentials are offered. The considered model connects a set of Maxwell's equations with a Schrödinger equation. On the basis of algorithm the program is developed in the Matlab.

Calculations for a particle of lithium were carried out and dependency graphs of the square of absolute values the electric vector amplitude from the frequency of an incident radiation are made. It is visible that in case of a particle with a radius of 1 nanometer the larger shift of a curve of the full electric vector from a curve of the electric vector considering only the transverse wave unlike a particle with a radius of 2 nanometers takes place. It can be bound to more strong influence of quantum properties on a particle of the smaller size. For a particle of lithium it is shown that the contribution of the interaction potentials near singular points of system (in this case $r=0$ ) is comparable to a contribution of the transverse electromagnetic field. When the incident 


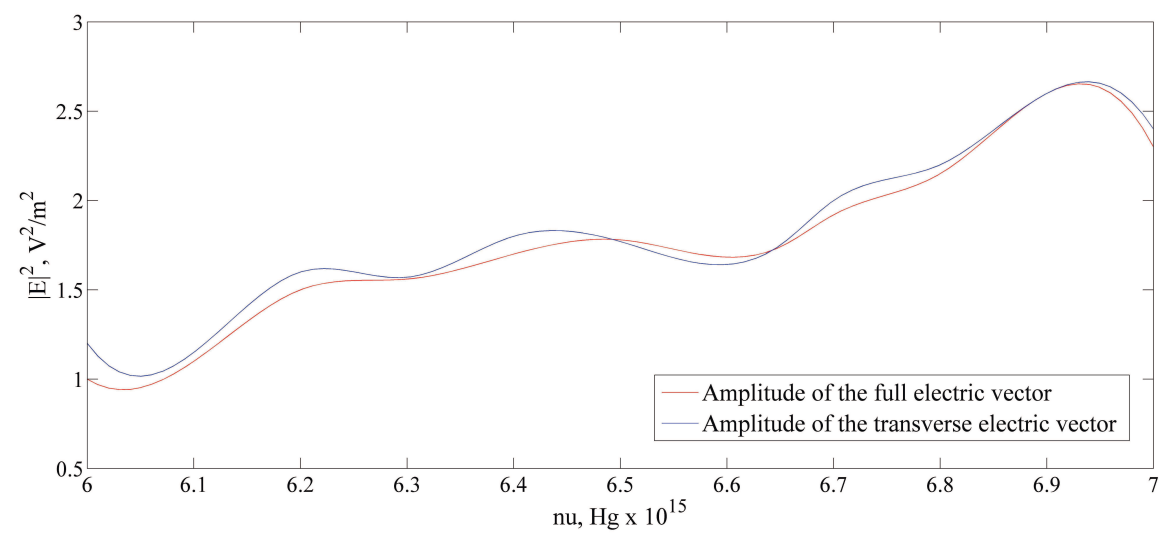

Figure 2: Comparison between the absolute values of the full electric vector amplitude and absolute values of the transverse electric vector amplitude with frequency of the incident radiation on the $\mathrm{x}$-axis (radius particle $\mathrm{R}=2 \mathrm{~nm})$. Direction of the ray along the radial coordinate

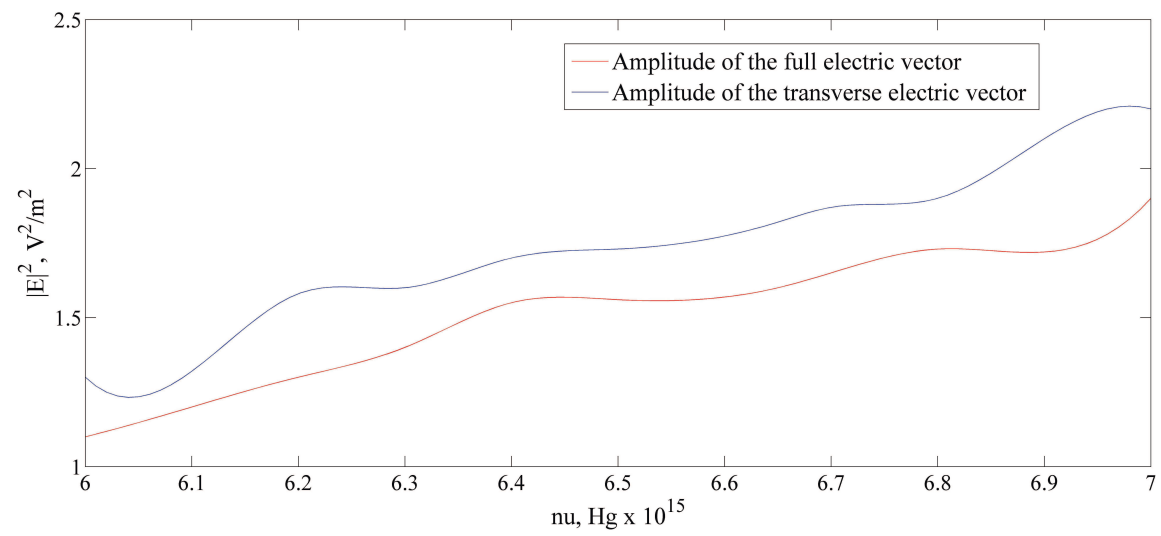

Figure 3: Comparison between the absolute values of the full electric vector amplitude and absolute values of the transverse electric vector amplitude with frequency of the incident radiation on the $\mathrm{x}$-axis (radius particle $\mathrm{R}=2 \mathrm{~nm})$. Direction of the ray along the radial coordinate

wave extends along the $z$-coordinate the contribution of interaction potentials dominates over the contribution of transverse electromagnetic field if non-linear parameter is positive. And versa vice if it is negative.

Thus, the values of the amplitude of longitudinal waves in nanosystems with 


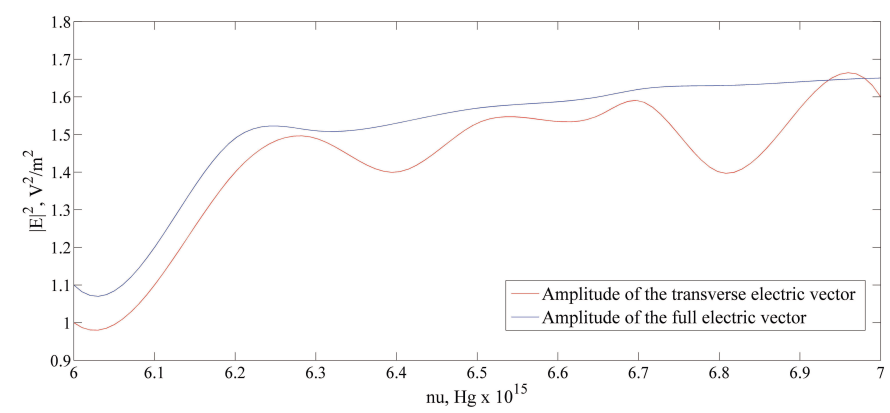

Figure 4: Comparison between the absolute values of the full electric vector amplitude and absolute values of the transverse electric vector amplitude with frequency of the incident radiation on the $\mathrm{x}$-axis (radius particle $\mathrm{R}=2 \mathrm{~nm}), \alpha>0$. Direction of the ray is along the coordinate $\mathrm{z}$.

cylindrical geometry depends on types of the dielectric permittivity as functions of the electric vector and the characteristic dimension of system, and on the direction of the rays under consideration.

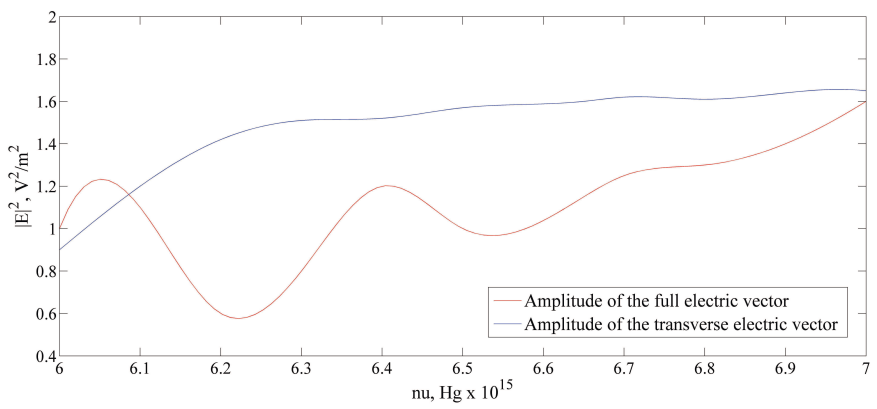

Figure 5: Comparison between the absolute values of the full electric vector amplitude and absolute values of the transverse electric vector amplitude with frequency of the incident radiation on the $\mathrm{x}$-axis (radius particle $\mathrm{R}=1 \mathrm{~nm}), \alpha>0$. Direction of the ray is along the coordinate z 


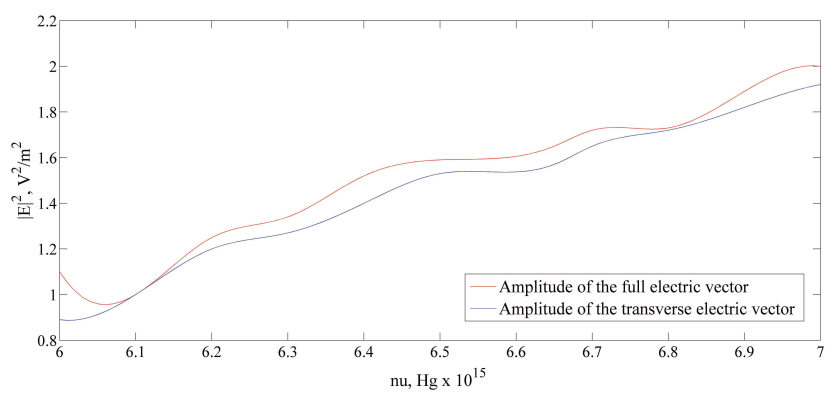

Figure 6: Comparison between the absolute values of the full electric vector amplitude and absolute values of the transverse electric vector amplitude with frequency of the incident radiation on the $\mathrm{x}$-axis (radius particle $\mathrm{R}=21 \mathrm{~nm}$ ), Direction of the ray is along the coordinate $\mathrm{z}$, $\alpha<0$.

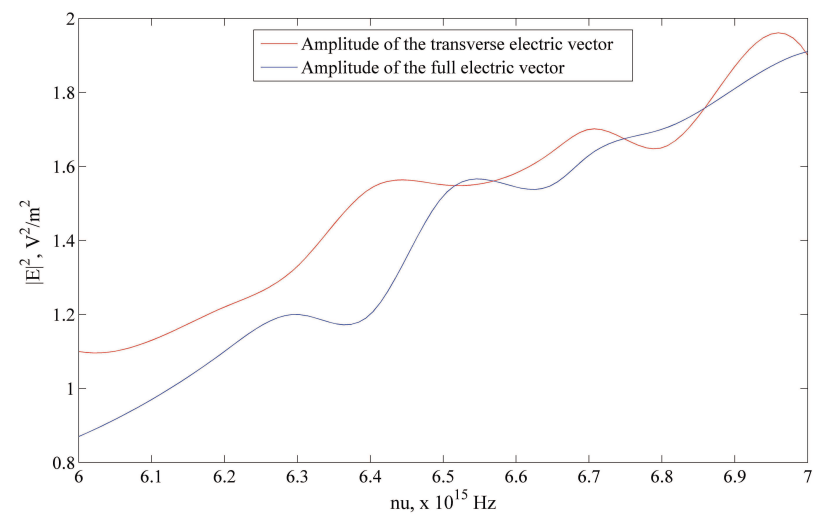

Figure 7: Comparison between the absolute values of the full electric vector amplitude and absolute values of the transverse electric vector amplitude with frequency of the incident radiation on the $\mathrm{x}$-axis (radius particle $\mathrm{R}=1 \mathrm{~nm}$ ), Direction of the ray is along the coordinate $\mathrm{z}, \alpha<0$

\section{Acknowledgments}

This work was supported by the Russian Foundation for Basic Research under grant No 15-01-08073 and Science of Russia in the framework of a state order in the sphere of scientific activities (order No. 2014/105, project No. 1441). 


\section{References}

[1] M. Homber, U. Simon, On the application potential of gold nanoparticles in nanoelectronics and biomedicine, Phil. Trans. R. Soc. A, 368, (2010), 1405-1453.

[2] M. Abhilash, Potential applications of Nanoparticles, International Journal of Pharma and Bio Sciences, 1, No.1, (2010), 1-12.

[3] J. Liu, Al. Levine, JS. Matton, M. Yamaguchi, RJ. Lee (et al.), Nanoparticles as image enhancing agents for ultrasonography, Physics in Medicine and Biology, 51, (2006), 21792189 .

[4] E. Araujo, N.J. Andrade, LHM. Da Silva, PC. Bernardes, AVNC. Teixeira (et al.), Antimicrobial effects of silver nanoparticles against bacterial cells adhered to stainless steel surfaces, Journal of Food Production, 74, No.4 (2011), 701-705.

[5] U. Kreibig, M. Vollmer, Optical properties of metal clusters, Springer series in material science, New York (1995).

[6] E. Lorin, S. Chelkowski, A. Bandrauk, A numerical Maxwell-Schrödinger model for intense laser-molecule interaction and propagatio, Computer Physics Communications, 177 (2007), 908-932.

[7] E. Lorin, S. Chelkowski, A. Bandrauk, AA MaxwellSchrödinger Model for Nonperturbative Laser-molecule Interaction and Some Methods of Numerical Computation, Centre de Recherches Mathématiques CRM Proceedings and Lecture Notes, 41 (1987), 908-932.

[8] L. A. Uvarova and V, K. Fedyanin, Asymptotic solutions for electromagnatic waves in a nonlinear optical cylinder Theoretical and Mathematical Physics, 106, No.1, (1996), 68-74.

[9] K. Bohren, D. Huffman, Absorption and Scattering of Light by Small Particles, Wiley Science Paperback Series, New York (1998).

[10] M. Born, E. Wolf, Electromagnetic Theory of Propagation, Interference and Diffraction of Light, Cambridge University Press, Cambridge (1999).

[11] A. D. Bandrauk Molecules in laser field, Marcel Dekker, New York (1994).

[12] D. R. Hartree, The calculation of atomic structure, John Wiley \& Sons, New York (1957).

[13] M. Brack, The physics of simple metal clusters: self-consistent jellium model and semiclassical approaches Rev. Mod. Phys., 65, No.3 (1993), 677-732.

[14] M. Born, R. Oppenheimer, Zur Quantentheorie der Molekeln Annalen der Physik, 389, No.20 (1927), 457-484.

[15] V. K. Ivanov, Electron properties of metallic clusters Soros Education Journal, 70, (1987), 323-341 (in Russian).

[16] R. G. Polozkov, V. K. Ivanov, A. V. Verkhovtsev, A. V. Solov'yov, Stability of metallic hollow cluster systems: Jellium model approach Phys. Rev. A, 79, (2009), 063203.

[17] V.K. Ivanov, A.N.Ipatov, V.A. Kharchenko, M. L. Zhyzhin, Electronic structure and adiabatic oscillation of metal-clusters JETP Letters, 8, No. 58, (1993), 649-655.

[18] J. C. Morrison, Modern Physics for Scientists and Engineers, Academic Press/Elsevier, London (2015).

[19] Ira N. Levine, Quantum Chemistry, Prentice Hall, New Jersey (2000). 
[20] T. Tsuneda, Density Functional Theory in Quantum Chemistry, Springer Japan, Tokyo (2014).

[21] D. Potter, Computational methods in Physics, John Wiley \& Sons, New York (1973). 